\title{
MODELAGEM DE ATRIBUTOS FÍSICO-HÍDRICOS DO SOLO NUMA BACIA HIDROGRÁFICA DA REGIÃO DO ALTO RIO GRANDE, MG $^{(1)}$
}

\author{
Carlos Rogério de Mello( ${ }^{(2)}$, Natalino Martins Gomes ${ }^{(3)}$, Antônio \\ Marciano da Silva ${ }^{(4)}$ \& José A. Junqueira Junior ${ }^{(5)}$
}

\begin{abstract}
RESUMO
O presente trabalho objetivou desenvolver modelos para estimativa dos atributos físico-hídricos do solo porosidade drenável (PD) e capacidade total de retenção de água (CTA), com base em atributos físicos de fácil e rápida obtenção (textura, argila dispersa em água, volume total de poros, densidade do solo e densidade de partícula) e a determinação de matéria orgânica. Os atributos foram coletados na bacia hidrográfica do Ribeirão Marcela, na camada de 0 a $15 \mathrm{~cm}$, obedecendo aos grids de 240 × 240 e 60 × $60 \mathrm{~m}$, totalizando 165 pontos amostrados. Trabalhou-se com regressão múltipla linear, constituindo-se variáveis por meio da combinação dos diferentes atributos entre si e estimando os respectivos coeficientes pelo método dos mínimos quadrados, utilizando-se o programa SAS for Windows. Os modelos gerados para CTA e PD apresentaram boa qualidade estatística, com elevados coeficientes de determinação, baixos erros de estimativa e variáveis significativas, o que permitiu sua aplicação na estimativa desses atributos na bacia hidrográfica do Ribeirão Marcela, representativa do domínio dos Latossolos na região do Alto Rio Grande, fornecendo assim subsídios para implantação de projetos agrícolas e ambientais, especialmente no auxílio à parametrização de modelos de simulação hidrossedimentológica, os quais estão em desenvolvimento para aplicação na região.
\end{abstract}

Termos de indexação: porosidade drenável, retenção de água no solo, Latossolos.

\footnotetext{
(1) Recebido para publicação em setembro de 2005 e aprovado em maio de 2007.

(2) Professor Adjunto do Departamento de Engenharia, Universidade Federal de Lavras - UFLA. Caixa Postal 37, CEP 37200-000 Lavras (MG). Bolsista CNPq. E-mail: crmello@ufla.br

(3) Doutorando em Engenharia Agrícola, UFLA. Bolsista CAPES. E-mail: natalagricola@yahoo.com.br

(4) Professor Titular, Universidade Federal de Lavras. Bolsista CNPq. E-mail: marciano@ufla.br

(5) Mestre em Engenharia Agrícola, UFLA. Bolsista CNPq. E-mail: junqueirajunior@yahoo.com.br
} 


\title{
SUMMARY: PHYSICAL-HYDRIC SOIL ATTRIBUTE MODELING IN A WATERSHED IN THE REGION OF ALTO RIO GRANDE, MG
}

\begin{abstract}
This study aimed to develop models to estimate the two physical-hydric soil attributes, drainable porosity (PD) and water holding capacity (CTA), based on easily and quickly measured physical attributes (particle-size distribution, water dispersible clay, total porosity, bulk density and particle density). Disturbed and undisturbed soil samples were collected in the Ribeirão Marcela watershed, from the of 0-15 cm layer, in grids of $240 \times 240$ and $60 \times 60 \mathrm{~m}$, totaling 165 sample points. Linear multiple regression was applied, using variables that combined the different attributes with each other and the coefficients of variables estimated by the Least-Squares Estimation, using the software SAS. The models for CTA and PD presented good statistical qualities, with high coefficients of determination, low estimate error and, statistical significance of estimated parameters. These results suggest that these models might be used for projects in the Ribeirão Marcela watershed, which is representative of the Oxisols domain in the Alto Rio Grande region. They further provide region-specific technical information for agricultural and environmental projects and above all, for the parameterization of the hydrosedimentological simulation models in development.
\end{abstract}

Index terms: drainable porosity, water holding capacity, Oxisols.

\section{INTRODUÇÃO}

A região do Alto Rio Grande, a montante do reservatório da Usina Hidrelétrica (UHE) de Camargos, é uma das regiões estratégicas, do ponto de vista energético, para o Estado de Minas Gerais, no século XXI. Por meio de dois rios principais, Grande e Aiuruoca, forma-se o reservatório da referida usina, o qual é responsável direto pela produção de sua energia, além de manter dois outros reservatórios a sua jusante, os quais são a "fio d'água", nas usinas de Itutinga e do Funil.

O manejo adequado dos solos da região é de fundamental importância para promoção de um desenvolvimento agropecuário sustentável, o qual vem crescendo nos últimos anos. A região pode ser dividida em três diferentes unidades de planejamento, sendo uma delas junto à Serra da Mantiqueira, próximo às principais nascentes da região, cuja aptidão está associada à proteção ambiental, especialmente perenização de cursos d'água; uma outra, sob domínio de Cambissolos de baixa fertilidade, utilizada basicamente para sustentar pastagem extensiva de gado; e a terceira, sob domínio dos Latossolos, mais próxima ao reservatório de Camargos, com aptidão para lavouras (Giarola et al., 1997). A bacia hidrográfica do Ribeirão Marcela, onde este estudo foi desenvolvido, é considerada representativa deste último ambiente, segundo estudos prévios desenvolvidos por Motta et al. (2001).

Ainda segundo Motta et al. (2001), esta bacia hidrográfica é constituída praticamente por Latossolos (80 \% de sua área), com aptidão agrícola para culturas anuais, sobretudo grãos, o que também foi constatado e reforçado pelo trabalho de Gomes (2005). Assim, espera-se que o manejo do solo para esse fim, especialmente com uso de irrigação, seja cada vez mais implementado, necessitando-se para isso de subsídios técnicos, principalmente relacionados à disponibilidade hídrica do solo.

A estimativa de atributos físico-hídricos de lenta e custosa obtenção no laboratório, por meio de modelos matemáticos, ajustados como função de atributos físicos de rápida e fácil determinação, como textura, matéria orgânica, densidade do solo e volume total de poros, tem sido uma alternativa para geração de informações técnicas para análise prévia da viabilidade técnico-econômica de projetos hidroagrícolas, como irrigação, assentamentos agrícolas e valoração da terra para posterior doação ou indenização por órgãos públicos e privados, no caso de projetos estratégicos de forte apelo ambiental, como inundação de áreas, aterros sanitários, linhas de transmissão, plantio de eucalipto para celulose - algo que vem crescendo muito na região, entre outros (Alvarenga \& Souza, 1998; Tomasella et al., 2000; Mello et al., 2002, 2005; Hodnett \& Tomasella, 2002). Ressalta-se que as variáveis de entrada desses modelos, citadas anteriormente, além de rotineiras em laboratórios, podem ser extraídas de levantamentos pedológicos, como os desenvolvidos por Giarola et al. (1997), Motta et al. (2001) e Curi et al. (2005).

Além dos aspectos mencionados no tocante ao manejo do solo, o desenvolvimento e aplicação de modelos hidrossedimentológicos para simulação dos escoamentos superficial e subterrâneo, bem como do transporte de sedimentos, tem sido ferramenta extremamente importante para previsão dos impactos produzidos por mudanças no uso do solo, alterando o ambiente natural da bacia hidrográfica. Nesse contexto, de acordo com Collischonn (2001) e Moraes et al. (2003), a estimativa de atributos físicos do solo torna-se fundamental para parametrização desses modelos hidrossedimentológicos, especialmente aqueles baseados no processo de sedimentação. 
Todo e qualquer estudo hidrológico e pedológico desenvolvido nessa bacia é de fundamental importância no contexto do manejo ambiental sustentável, e seus resultados poderão ser aplicados na região mais próxima ao reservatório da UHE de Camargos/CEMIG, sendo esta promissora no contexto tanto agropecuário quanto energético do sul de Minas Gerais. Portanto, este trabalho objetivou gerar modelos para estimativa dos atributos físico-hídricos do solo porosidade drenável (PD) e capacidade total de retenção de água (CTA), baseados em atributos físicos de fácil e rápida obtenção, para uma bacia hidrográfica representativa do domínio dos Latossolos na região Alto Rio Grande, a montante do reservatório da UHE de Camargos/ CEMIG.

\section{MATERIAL E MÉTODOS}

A bacia hidrográfica do Ribeirão Marcela encontra-se entre as coordenadas UTM “550169" e "552810" de longitude W e "7650163" e "7650989" de latitude S e entre as altitudes de 960 e $1.060 \mathrm{~m}$. Está inserida na unidade geomorfológica Planalto Campos das Vertentes, na região hidrográfica do Alto Rio Grande. Esta bacia hidrográfica é representativa dos ambientes associados ao Latossolo na região Alto Rio Grande, segun- do Motta et al. (2001). Além disso, essa representatividade está embasada em estudo hidrogeológico recente, realizado por Bertachini et al. (2005), os quais destacam essa bacia com boas condições para recarga dos aqüíferos subterrâneos.

Caracterizada com o relevo suave ondulado a ondulado e clima Cwa pela classificação de Köppen, possui área de 477 ha, drenando para o Ribeirão Jaguara, o qual drena diretamente para o reservatório da UHE de Camargos/CEMIG. A distribuição das unidades pedológicas na bacia hidrográfica apresenta-se da seguinte forma: Latossolo Vermelho-Amarelo distrófico (LVAd), ocupando $65 \%$ da área; Latossolo Vermelho distrófico típico (LEd), em 14 \% da área; Cambissolos (C), em menos de $5 \%$; e Solos Hidromórficos (Hi), em $17 \%$ da área da bacia.

Foi realizada amostragem sistemática ao longo da bacia hidrográfica, obedecendo-se a um grid quadrado de $240 \mathrm{~m}$ de lado, com adensamento desta em quatro áreas consideradas importantes do ponto de vista de uso do solo, em que o grid foi subdividido em outro grid de $60 \mathrm{~m}$ de lado. O total de informações trabalhadas foi de 165 pontos na camada de 0 a $15 \mathrm{~cm}$, coletando-se amostras deformadas e não-deformadas. $\mathrm{Na}$ figura 1 encontra-se o mapa de solos da bacia hidrográfica, com a distribuição dos pontos amostrados.

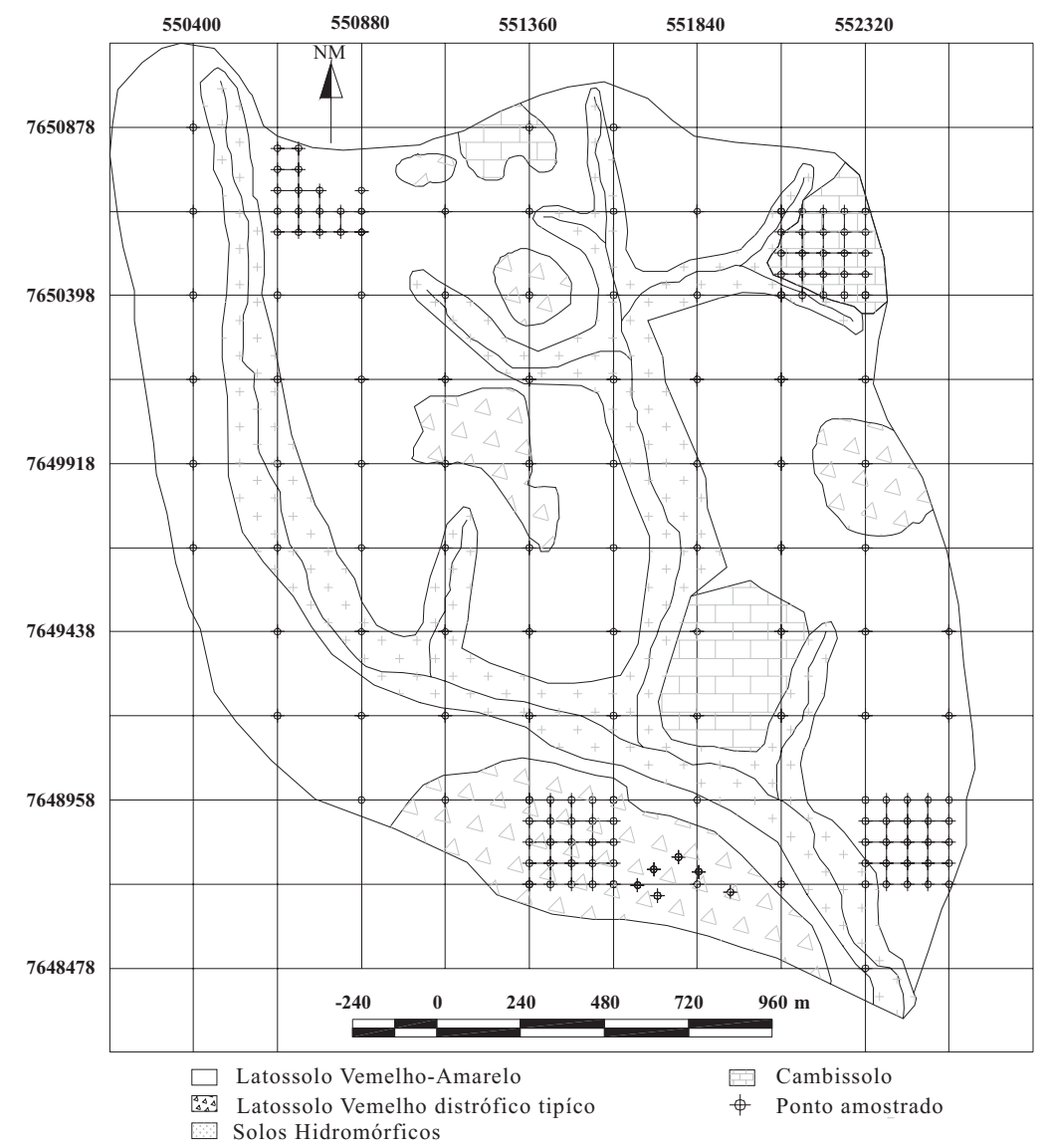

Figura 1. Unidades pedológicas presentes na bacia hidrográfica do Ribeirão Marcela e esquema de amostragem sistemática utilizada nos trabalhos de campo. 
Foram feitas análises físicas de textura (areia, silte, argila) pelo método da pipeta (Embrapa, 1997); densidade do solo (DS), pelo método do anel volumétrico (Embrapa, 1997); densidade de partículas (DP), pelo método do picnômetro; argila dispersa em água (ADA), pelo método da pipeta, sem adição de $\mathrm{NaOH}$; e matéria orgânica obtida do carbono orgânico, multiplicando-o por 1,724 (Walkley \& Black, 1934). O volume total de poros $\left(\mathrm{VTP}_{\mathrm{c}}\right)$ foi obtido pela expressão:

$$
\mathrm{VTP}_{\mathrm{C}}=100 \cdot\left(1-\frac{\mathrm{D}_{\mathrm{s}}}{\mathrm{D}_{\mathrm{p}}}\right)
$$

A Capacidade Total de Retenção de Água (CTA, em $\mathrm{mm}$ ) foi determinada com base na diferença entre a umidade volumétrica na capacidade de campo ( $\theta c c)$ e a umidade volumétrica no ponto de murcha permanente $\left(\theta_{\mathrm{PMP}}\right)$, multiplicada pela profundidade de coleta - no caso, $150 \mathrm{~mm}$, sendo a primeira umidade estimada com base na tensão de $10 \mathrm{kPa}$ (Medina \& Oliveira Jr., 1987) e a segunda, a $1.500 \mathrm{kPa}$ (Reichardt, 1988):

$$
\mathrm{CTA}=\left(\theta_{\mathrm{CC}}-\theta_{\mathrm{PMP}}\right) 150
$$

A porosidade drenável ( $\mathrm{PD}$, em $\mathrm{m}^{3} \mathrm{~m}^{-3}$ ) foi calculada com base na umidade volumétrica de saturação $\left(\theta_{\text {sat }}\right)$ e $\theta c c$, conforme Queiroz (1995) e Mello et al. (2002), e aplicando-se a expressão:

$$
\mathrm{PD}=\left(\theta_{\mathrm{sat}}-\theta_{\mathrm{CC}}\right)
$$

Os modelos para CTA e PD foram desenvolvidos em função dos atributos físicos, a saber: percentagens de areia, silte, argila e matéria orgânica (MO), argila dispersa em água (ADA), VTP, DS e DP. Assim, trabalhou-se com regressão múltipla linear, constituindo-se variáveis por meio da combinação dos diferentes atributos entre si e estimando os respectivos coeficientes pelo método dos mínimos quadrados. Por intermédio do procedimento Backward, essas variáveis foram selecionadas em função de sua respectiva significância estatística, sendo eliminadas aquelas nãosignificativas em nível de probabilidade máximo de $10 \%$ pelo teste $t$ de Student (Prob $>|t|$ máxima de $10 \%)$. Todo o processo de modelagem foi realizado utilizando-se o programa SAS for Windows, na rotina "ProcReg" (SAS, 1985). Na análise da acurácia dos modelos, além das significâncias das variáveis, foram considerados o coeficiente de determinação $\left(\mathrm{R}^{2}\right)$, os erros médio e máximo obtidos pelas estimativas dos modelos em relação ao valor observado, a dispersão dos valores em torno da reta 1:1 e a dispersão das diferenças entre os valores observados e estimados em relação aos observados.

\section{RESULTADOS E DISCUSSÃO}

No quadro 1 apresentam-se algumas estatísticas básicas dos atributos físicos dos solos da bacia hidrográfica, as quais refletem o comportamento da variabilidade dos dados trabalhados.

Analisando o coeficiente de variação e tomando-se como base os critérios propostos por Warrick \& Nielsen (1980), que consideram os valores deste coeficiente entre 12 e $60 \%$ como de média variabilidade e os valores abaixo e acima deste intervalo como de baixa e alta variabilidade, respectivamente, percebe-se variabilidade baixa a média para os atributos, sendo ADA o que produziu o maior coeficiente de variação: 57 \%. É interessante mencionar a predominância da fração argila e altos valores para VTP, sendo ambas as características típicas dos Latossolos da região, favorecendo a implantação de lavouras anuais. Ressalta-se também que o valor médio da DS está

Quadro 1. Estatísticas básicas dos atributos físico-hídricos do solo da bacia hidrográfica do Ribeirão Marcela, região Alto Rio Grande, MG

\begin{tabular}{lcccc}
\hline Atributo & Valor mínimo & Valor médio & Valor máximo & CV (\%) \\
\hline DS $\left(\mathrm{kg} \mathrm{dm}^{-3}\right)$ & 0,83 & 1,12 & 1,32 & 9,19 \\
DP $\left(\mathrm{kg} \mathrm{dm}^{-3}\right)$ & 1,92 & 2,53 & 2,70 & 3,28 \\
Areia (\%) & 9,00 & 20,96 & 56,00 & 33,04 \\
Argila (\%) & 25,00 & 60,02 & 82,00 & 33,36 \\
MO $\left(\mathrm{dag} \mathrm{kg}^{-1}\right)$ & 1,00 & 2,83 & 5,30 \\
VTP $\left(\mathrm{m}^{3} \mathrm{~m}^{-3}\right)$ & 0,425 & 0,559 & 0,679 & 57,86 \\
ADA $(\%)$ & 0,00 & 8,46 & 31,00 & 43,51 \\
PD $\left(\mathrm{m}^{3} \mathrm{~m}^{-3}\right)$ & 0,043 & 0,154 & 0,326 & 23,51 \\
CTA $\left(\mathrm{mm}^{2}\right.$ & 11,123 & 25,086 & 39,264 & \\
\hline
\end{tabular}


condizente com seus valores típicos, os quais são influenciados pelas características mineralógicas e texturais do material de origem. No entanto, foram encontrados valores da ordem de $1,3 \mathrm{~kg} \mathrm{dm}^{-3}$, o qual é considerado como acima do valor normal esperado, podendo significar algum processo de compactação na camada superficial do solo, causada por excesso de pastejo, segundo Gomes (2005), o qual avaliou o uso atual dos solos da bacia hidrográfica. Conclui-se que a densidade de partículas (DP) é o atributo que apresenta menor variabilidade, com pequena oscilação entre os valores mínimo e máximo, conforme já consagrado na literatura. Como DS e DP possuem pequena variabilidade, o volume total de poros (VTP) também produziu esse mesmo comportamento, visto que foi calculado em função desses atributos. Quanto aos atributos físico-hídricos modelados, a porosidade drenável (PD) apresentou coeficiente de variação de $43,51 \%$, caracterizando variabilidade média dos dados. Da mesma forma, para CTA, obteve-se coeficiente de variação de $23,51 \%$, valor este consideravelmente menor que o obtido para PD, mostrando menor variabilidade, mas, ainda sim, caracterizado por média variabilidade, de acordo com o critério de Warrick \& Nielsen (1980), adotado neste trabalho.

Nos quadros 2 e 3 são apresentados, respectivamente, os modelos ajustados para CTA e PD, bem como os respectivos coeficientes de determinação e erros médio e máximo. No modelo para CTA, observa-se maior quantidade de variáveis em relação ao modelo para PD. Isso significa que houve maior dificuldade para obtenção final do modelo, produzindo,

Quadro 2. Variáveis constituintes, respectivos parâmetros estimados e significância pelo teste t de Student para o modelo ajustado para CTA

\begin{tabular}{|c|c|c|}
\hline Variável & Parâmetro estimado & Prob $>|\mathbf{t}|$ \\
\hline Intercepto & 1532,901 & $0,0895^{\square}$ \\
\hline DS & $-4415,706$ & $0,0001^{* *}$ \\
\hline Areia & 31,907 & $0,0001 * *$ \\
\hline Argila & 12,997 & $0,0115^{*}$ \\
\hline MO & 80,036 & $0,0001 * *$ \\
\hline VTP & $-4423,889$ & $0,1365^{\mathrm{ns}}$ \\
\hline $\mathrm{ADA}$ & 5,574 & $0,0026^{* *}$ \\
\hline Areia $^{2}$ & 0,4409 & $0,2177^{\mathrm{ns}}$ \\
\hline $\mathrm{MO}^{2}$ & $-5,187$ & $0,0001 * *$ \\
\hline $\mathrm{DS}^{2}$ & 4214,169 & $0,0001^{* *}$ \\
\hline $\mathrm{ADA}^{2}$ & 0,02289 & $0,0927^{\square}$ \\
\hline $\mathrm{VTP}^{2}$ & 1,0862 & $0,0421^{*}$ \\
\hline Areia $\mathrm{x}$ argila & $-0,3238$ & $0,0001^{* *}$ \\
\hline Areia x MO & $-1,9129$ & $0,0001 * *$ \\
\hline Areia x DS & $-4,681$ & $0,019^{*}$ \\
\hline Areia x ADA & $-0,0843$ & $0,0008^{* *}$ \\
\hline Argila x MO & $-0,8326$ & $0,0004^{* *}$ \\
\hline Argila x DS & $-2,028$ & $0,2943^{\mathrm{ns}}$ \\
\hline Argila x ADA & $-0,1019$ & $0,0001 * *$ \\
\hline MO x DS & 9,928 & $0,0223^{*}$ \\
\hline $\mathrm{MO} \times \mathrm{ADA}$ & 1,0631 & $0,0001 * *$ \\
\hline Areia $\mathrm{x}$ argila $\mathrm{x}$ VTP & 0,6316 & $0,0001^{* *}$ \\
\hline Areia x VTP & $-43,298$ & $0,0001 * *$ \\
\hline Argila x VTP & $-15,329$ & $0,0044^{* *}$ \\
\hline Areia $^{3}$ & $-0,000904$ & $0,0265^{*}$ \\
\hline $\mathrm{VTP}^{3}$ & $-6965,94$ & $0,0305^{*}$ \\
\hline $\mathrm{DS}^{3}$ & $-1264,696$ & $0,0001^{* *}$ \\
\hline Areia $^{2} \times \mathrm{MO}^{2}$ & 0,00451 & $0,0035^{* *}$ \\
\hline $\operatorname{Argila}^{2} \times \mathrm{MO}^{2}$ & 0,000581 & $0,0331^{*}$ \\
\hline $\mathrm{MO}^{2} \times \mathrm{ADA}^{2}$ & $-0,004861$ & $0,0035^{* *}$ \\
\hline $\mathrm{R}^{2}$ & 0,8311 & \\
\hline Erro médio (\%) & 6,86 & \\
\hline Erro máximo (\%) & 30,94 & \\
\hline
\end{tabular}

ns: Não-significativo; $\square$ : Significativo a $10 \%$; *: Significativo a $5 \%$; ** Significativo a $1 \%$. MO: matéria orgânica; DS: densidade do solo; ADA: argila dispersa em água; VTP: volume total de poros. 
entretanto, acurácia um pouco maior que o modelo para $\mathrm{PD}$, com menores erros, apesar do coeficiente de determinação de mesma magnitude. Mello et al. (2002) também encontraram dificuldade e resultados semelhantes trabalhando com ajuste de modelos lineares para predizer CTA e PD essencialmente para Cambissolos, na mesma região deste estudo. No entanto, a quantidade de informações trabalhadas pelos autores é substancialmente menor, com conseqüências negativas para o grau de liberdade dos modelos.

É interessante destacar que os atributos texturais, bem como o teor de matéria orgânica, predominam nos ajustes, demonstrando a importância destes na determinação das características físico-hídricas do solo, inclusive para os Latossolos. Saxton et al. (1986) ajustaram modelos com boas qualidades estatísticas para as umidades volumétricas na capacidade de campo e ponto de murcha permanente em função da textura, trabalhando com solos de clima temperado, onde, de maneira geral, a textura explica praticamente todo o comportamento físico-hídrico do solo. No caso do presente estudo, além do teor de matéria orgânica, foi possível constatar forte influência da densidade do solo e do volume total de poros, os quais são importantes especialmente em termos da estrutura do solo, além de suscetíveis ao manejo, o que, no caso de solos intemperizados, a exemplo dos Latossolos, tem peso considerável no comportamento de atributos físico-hídricos, como é o caso da CTA e PD.
Especificamente em termos da acurácia dos modelos, observa-se que os coeficientes de determinação são consideravelmente elevados em se tratando de modelos para estimativa de atributos físico-hídricos, além de erros médios e máximos de pequena magnitude, especialmente para CTA. Saxton et al. (1986), Zhuang et al. (2001) e Mello et al. (2002, 2005), trabalhando com ajuste de modelos baseados em atributos texturais e teor de matéria orgânica, obtiveram acurácia semelhante, com coeficiente de determinação variando de 0,65 a 0,90 , concluindo sobre a boa acurácia destes, bem como suas qualidades, para estimativa dos atributos físico-hídricos a que se propõem.

Há três variáveis cujos parâmetros estimados são não-significativos (VTP, areia ${ }^{2}$ e argila x DS), mas que foram mantidos no modelo. Apesar de não apresentarem significância, essas variáveis estão colaborando para um coeficiente de determinação mais alto, e, se elas fossem excluídas, este seria reduzido. Como os graus de liberdade do modelo são altos, não há inconveniente em manter tais variáveis, uma vez que elas influenciam positivamente a qualidade final do modelo (Mello, 2003).

É possível observar também que os atributos silte e DP não foram incorporados aos modelos. O primeiro, por se constituir em co-variável, uma vez que é obtido por diferença, sendo função dos percentuais de areia e

\section{Quadro 3. Variáveis constituintes, respectivos parâmetros estimados e significância pelo teste t de Student} para o modelo ajustado para $\mathrm{PD}$

\begin{tabular}{lcl}
\hline Variável & Parâmetro estimado & Prob $>|\mathbf{t}|$ \\
\hline Intercepto & $-3,408$ & $0,0301^{*}$ \\
Areia & 0,05997 & $0,0007^{* *}$ \\
DS & 8,694 & $0,0487^{*}$ \\
Areia & & $0,0009^{* *}$ \\
MO $^{2}$ & $-0,000566$ & $0,0001^{* *}$ \\
DS $^{2}$ & 0,003266 & $0,0513^{\square}$ \\
VTP $^{2}$ & $-8,0775$ & $0,0001^{* *}$ \\
Areia x argila & 1,6628 & $0,0001^{* *}$ \\
Argila x ADA & $-0,000103$ & $0,0015^{* *}$ \\
MO x ADA $^{*}$ & 0,000076935 & $0,0003^{* *}$ \\
Areia x VTP & $-0,001911$ & $0,0036^{* *}$ \\
Areia & \\
DS VTP & & $0,0054^{* *}$ \\
R $^{2}$ & $-0,09581$ & $0,0602^{\square}$ \\
Erro médio (\%) & 0,001762 & \\
Erro máximo (\%) & 2,4249 &
\end{tabular}

$\square$ : Significativo a $10 \%$; *: Significativo a $5 \%$; **: Significativo a $1 \%$. MO: matéria orgânica; DS: densidade do solo; ADA: argila dispersa em água; VTP: volume total de poros. 
argila, o que foi detectado e excluído pelo procedimento Backward. Todas as variáveis associadas à $\mathrm{PD}$ não foram significativas pelo teste $t$, sendo excluídas do processo sem comprometimento do coeficiente de determinação. Uma possível explicação para esse comportamento está relacionada ao fato de que esse atributo é influenciado quase que exclusivamente pela mineralogia do solo, não havendo relação com os diferentes manejos do solo na bacia hidrográfica, o que produz baixa variabilidade e, portanto, pouco explica o comportamento de atributos associados à umidade do solo, normalmente com alta variabilidade natural, fruto dos diferentes uso e manejo do solo.

Na figura 2 apresentam-se as dispersões dos valores estimados para CTA e PD pelos modelos ajustados, em torno da reta 1:1. É possível verificar pequena dispersão dos valores, mostrando que os erros de estimativa são baixos, reforçando os comentários anteriores. No entanto, é interessante destacar que, para CTA, verifica-se menor dispersão dos valores em relação ao modelo para $\mathrm{PD}$, demonstrando a acurácia ligeiramente superior do modelo para CTA. Constatase também concentração dos valores entre 20 e $30 \mathrm{~mm}$ para CTA, verificando-se, principalmente, poucos valores abaixo de $20 \mathrm{~mm}$. Para PD não se observa uma faixa muito específica de valores, notando-se, sobretudo, poucos valores acima de $0,25 \mathrm{~m}^{3} \mathrm{~m}^{-3}$.
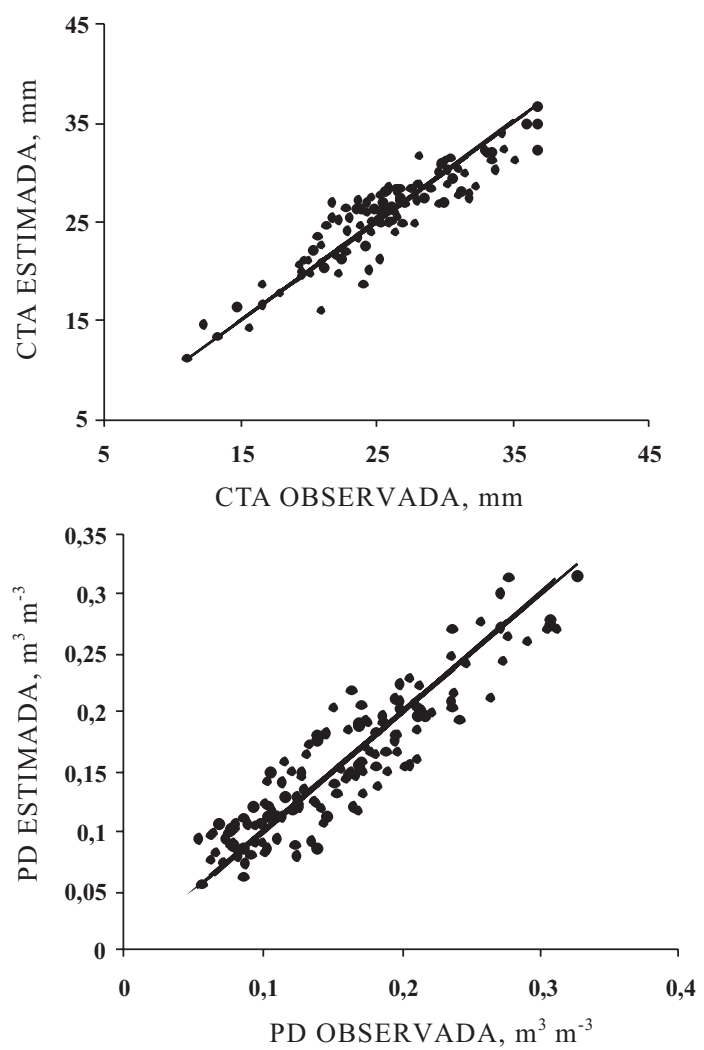

Figura 2. Dispersão dos valores estimados pelos modelos para CTA e PD em torno da reta 1:1.
Pela figura 3, a qual representa as diferenças entre os valores observados e estimados em função dos valores observados, é possível extrair alguns comportamentos importantes dos modelos. Para CTA, observa-se que o desvio máximo obtido, tanto para cima quanto para baixo, é menor que $6 \mathrm{~mm}$, com elevada concentração dos desvios entre - 2 e $2 \mathrm{~mm}$, com o erro mais provável estando nesse intervalo; verificam-se, portanto, pequenos erros de estimativa do modelo. No caso da PD, os desvios máximos são pouco menores que $0,06 \mathrm{~m}^{3} \mathrm{~m}^{-3}$. Da mesma forma que para CTA, o erro mais provável estará no intervalo de -0,03 e $0,03 \mathrm{~m}^{3} \mathrm{~m}^{-3}$, uma vez que há nele elevada concentração dos valores. Nessa análise, também se observa maior acurácia do modelo para CTA, pois o erro mais provável está dentro de um intervalo relativamente menor que aquele obtido para $\mathrm{PD}$, o que, no entanto, representa boa acurácia do modelo.

\section{CONCLUSÃO}

Os modelos de regressão múltipla linear gerados estimaram com boa acurácia os atributos físicohídricos do solo porosidade drenável e capacidade total de retenção de água, permitindo sua aplicação em estimativa desses atributos na bacia hidrográfica do Ribeirão Marcela, na região do Alto Rio Grande, MG.
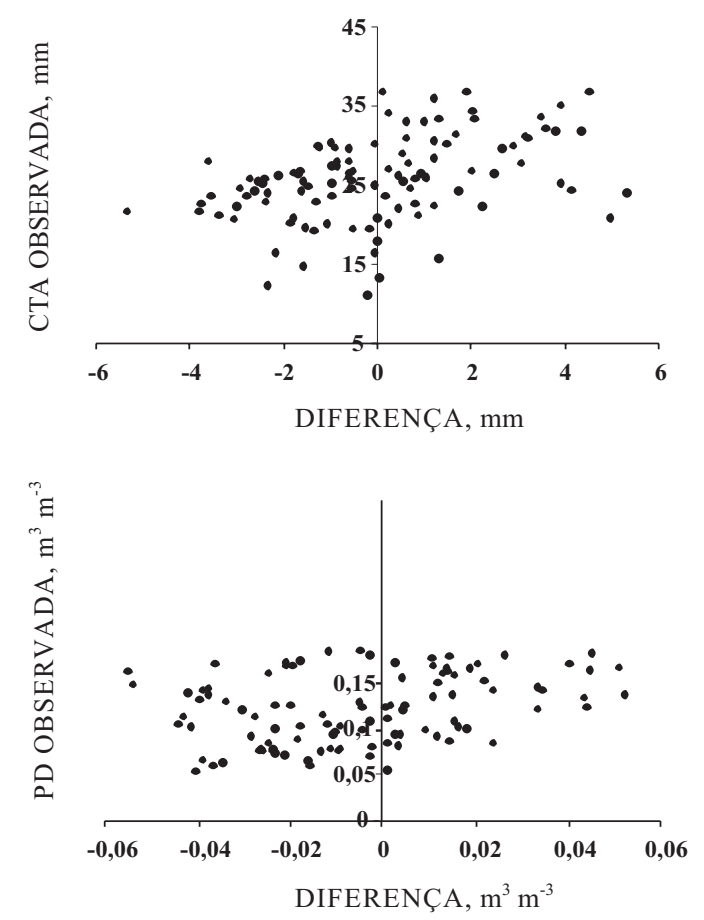

Figura 3. Comportamento das diferenças entre valores observados e estimados pelos modelos para CTA e PD para os solos da bacia hidrográfica do Ribeirão Marcela. 


\section{LITERATURA CITADA}

ALVARENGA, M.J.N. \& SOUZA, J.A. Atributos do solo e impacto ambiental. Lavras, Universidade Federal de Lavras, 1998. 205p.

BERTACHINI, A.C.; CANTARUTTI, C.C. \& DUTRA, G.M. Estudos hidrogeológicos sobre o regime de produção de água das bacias de drenagem de cabeceira da bacia do Rio Grande. In: SILVA, A.M.; MELLO, C.R. \& CORREA, M.R. Estudos hidrológicos sobre o regime de escoamento das bacias de drenagem do Alto Rio Grande a montante do reservatório da UHE-Camargos/CEMIG. Lavras, Belo Horizonte, UFLA/CEMIG/ANEEL，2005. p.91-114 (Relatório Parcial do Projeto de P\&D 076, CEMIG/ANEEL)

COLLISCHONN, W. Simulação hidrológica de grandes bacias. Porto Alegre, Universidade Federal do Rio Grande do Sul, 2001. 194p. (Tese de Doutorado)

CURI, N.; ARAUJO, A.R. \& MARQUES, J.J.G.S.M. Levantamento dos solos da bacia do Alto Rio Grande. In: SILVA, A.M.; MELLO, C.R. \& CORREA, M.R. Estudos hidrológicos sobre o regime de escoamento das bacias de drenagem do Alto Rio Grande a montante do reservatório da UHE-Camargos/CEMIG. Lavras, Belo Horizonte, UFLA/CEMIG/ANEEL, 2005. p.1-90 (Relatório Parcial do Projeto de P\&D 076, CEMIG/ANEEL)

EMPRESA BRASILEIRA DE PESQUISA AGROPECUÁRIA EMBRAPA. Centro Nacional de Pesquisa de Solos. Manual de métodos de análise de solo. 2.ed. Rio de Janeiro, Ministério da Agricultura e Abastecimento, 1997. 212p.

GIAROLA, M.F.B.; CURI, N.; SIQUEIRA, J.O.; CHAGAS, C.S. \& FERREIRA, M.M. Solos da região sob influência do reservatório da hidrelétrica de Itutinga/Camargos (MG): Perspectiva ambiental. Lavras, CEMIG/UFLA/FAEPE, 1997. 101p.

GOMES, N.M. Variabilidade espacial de atributos físico-hídricos do solo da sub-bacia hidrográfica do Ribeirão Marcela na região do Alto Rio Grande, MG. Lavras, Universidade Federal de Lavras, 2005. 124p. (Tese de Mestrado)

HODNETT, M.G. \& TOMASELLA, J. Marked differences between van Genuchten soil water-retention parameters for temperate and tropical soils: A new water-retention pedo-transfer functions developed for tropical soils. Geoderma, 108:155-180, 2002.

MEDINA, P.F. \& OLIVEIRA JR., R.C. Relações entre capacidade de campo determinada in situ e em laboratório em Latossolo Amarelo muito argiloso. R. Bras. Ci. Solo, 11:91-95, 1987.

MELLO, C.R. Estudo hidrológico em microbacia hidrográfica com regime de escoamento efêmero. Lavras, Universidade Federal de Lavras, 2003. 133p. (Tese de Doutorado)
MELLO, C.R.; OLIVEIRA, G.C.; FERREIRA, D.F.; LIMA, J.M. \& LOPES, D. Modelos para determinação dos parâmetros da equação de van Genuchten para um Cambissolo. R. Bras. Eng. Agric. Amb., 9:23-29, 2005.

MELLO, C.R.; OLIVEIRA, G.C.; FERREIRA, D.F. \& LIMA, J.M. Predição da porosidade drenável e disponibilidade de água para Cambissolos da Microrregião Campos das Vertentes, MG. Pesq. Agropec. Bras., 37:1319-1324, 2002.

MORAES, J.M.; SCHULER, A.E.; GUANDIQUE, M.E.G.; MILDE, L.C.; GROPPO, J.D.; MARTINELLI, A.L. \& VICTORIA, R.L. Propriedades físicas dos solos na parametrização de um modelo hidrológico. R. Bras. Rec. Hídricos, 8:61-70, 2003.

MOTTA, P.E.F.; CURI, N.; SILVA, M.L.N.; MARQUES, J.J.G.S.M.; PRADO, N.J.S. \& FONSECA, E.M.B. Levantamento pedológico detalhado, erosão dos solos, uso atual e aptidão agrícola das terras de microbacia piloto na região sob influência do reservatório da Hidrelétrica de Itutinga/Camargos-MG. Lavras, UFLA/CEMIG, 2001. 51p.

QUEIROZ, J.E. Parâmetros hidro-dinâmicos de um solo de várzea para fins de drenagem subterrânea. Piracicaba, Escola Superior de Agricultura Luiz de Queiroz, 1995. 167p. (Tese de Doutorado)

REICHARDT, K. Capacidade de campo. R. Bras. Ci. Solo, 12:211-216, 1988

SAS INSTITUDE. Statistical Analysis System: Language guide for personal computers. 6.ed. Cary, 1985. 429p.

SAXTON, K.E.; RAWLS, W.J.; ROMBERGER, J.S. \& PAPENDICK, R.I. Estimating generalized soil-water characteristics from texture. Soil Sci. Soc. Am. J., 50:10311036,1986

TOMASELLA, J.; HODNETT, M.G. \& ROSSATO, L. Pedotransfer functions for the estimation of soil water retention in Brazilian soils. Soil Sci. Soc. Am. J., 64:327338,2000

WALKLEY, A. \& BLACK, I.A. An examination of the Degtjareff method for determining soil organic matter and a proposed modification of the chromic acid titration method. Soil Sci., 37:29-38, 1934.

WARRICK, A.W. \& NIELSEN, D.R. Spatial variability of soil physical properties in the field. In: HILLEL, D., ed. Applications of soil physics. New York, Academic Press, 1980. p.319-344.

ZHUANG, J.; JIN, Y. \& MIYAZAKI, T. Estimating water retention characteristic from soil particle-size distribution using a non-similar media concept. Soil Sci., 166:308-321, 2001. 